\title{
OPTICAL PROPERTIES OF INTERPLANETARY DUST IN THE TANGENTIAL PLANE
}

\author{
J.B. RENARD, A.C. LEVASSEUR-REGOURD \\ Université PARIS 6 / Service d'Aéronomie - BP 3 \\ 91371 Verrières le Buisson, France
}

\author{
R. DUMONT \\ Observatoire de Bordeaux \\ 33270 Floirac, France
}

\begin{abstract}
Local intensity and emissivity, and consequently local polarization degree, temperature and albedo, can be retrieved from optical and thermal observations of zodiacal light. The local polarization degree (normalized at constant solar distance and phase angle) is found to decrease with elevation above the symmetry plane of the zodiacal cloud. The heterogeneity of the cloud, established towards the symmetry pole, is here demonstrated in the tangential plane (almost perpendicular to the ecliptic plane at $1 \mathrm{AU}$ ). We present a map of the local polarization degree in this plane.
\end{abstract}

\section{Introduction}

Measurements of light, either scattered or emitted by interplanetary dust, provide brightness integrals along the line of sight which extends from the observer to the outer fringe of the zodiacal cloud. Local contribution of the grains can be obtained by some local inversion. In order to perform an inversion, mathematical smooth functions (exponential, lorentzian, trigonometric, polynomial, Fourier series) which represent the elemental contributions, can be searched. The integral of these functions has to be equal to the measured brightnesses. The functions need also to satisfy various constraints because of the physical properties of the zodiacal cloud : to be monotonous, to be equal to zero at infinity, to decrease asymptotically to zero with increasing solar distance. It has been found that the curves representing the functions constrict in some nodal regions (fig.1) where the local contributions are determined with lesser uncertainties than elsewhere (Dumont, 1983; Dumont \& Levasseur-Regourd, 1985, 1988; Levasseur-Regourd \& Dumont, 1990).

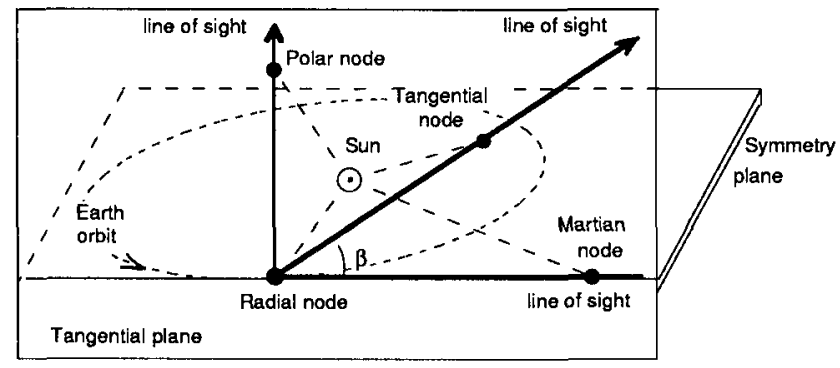

Figure 1: Geometry of the method.

A.C. Levasseur-Regourd and H. Hasegawa (eds.), Origin and Evolution of Interplanetary Dust, 199-202. (C) 1991 Kluwer Academic Publishers, Printed in Japan. 


\section{Presentation of the Method}

\subsection{INVERSION TECHNIQUES}

In the symmetry plane of the zodiacal cloud, two integrated brightnesses are available along the same line of sight which intersects the Earth's orbit for solar elongations $\varepsilon$ and $180^{\circ}-\varepsilon$. Curves which represent the functions for the local brightnesses have to focus in two nodal regions : the first one, called radial node, is located in the middle of the chord intersecting the Earth's orbit; the second one, called martian node, is located at a constant solar distance $\mathrm{R}=1.54 \mathrm{AU}$. Towards the pole of the symmetry plane, only one integrated brightness is available. So another data is required : the local intensity at $\alpha=90^{\circ}$ and $\mathrm{R}=1 \mathrm{AU}$; this result is obtained from the radial node value at an elongation of $90^{\circ}$. Curves have to focus in a nodal region called polar node (fig.2), at an elevation $h$ above the symmetry plane (Levasseur-Regourd et al.,1990). Using this method for integrated intensity ( 2 polarized components), integrated emissivity (at 2 wavelengths), and for polarimetry and temperature results, four polar node positions are obtained with an elevation varying from 0.2 to $0.4 \mathrm{AU}$.

We present here an extension of the method previously described, which allows us to derive local values at various ecliptic latitudes from available measurements in the tangential plane (tangent to the Earth's orbit and perpendicular to the symmetry plane). Curves representing the functions have to focus in a nodal region called tangential node, at an elevation varying from 0 to $0.4 \mathrm{AU}$ with ecliptic latitude $\beta$ increasing from $0^{\circ}$ to $90^{\circ}$. Using this method to available intensity data (by steps of $5^{\circ}$ in $\beta$ ), two tangential node positions are obtained for each line of sight.

The method developed in the symmetry plane for $\varepsilon=90^{\circ} \mathrm{can}$ also be applied in the tangential plane. One additional tangential node is derived on each line of sight for $\beta$ smaller than $15^{\circ}$. Its elevation remains between 0 and $0.3 \mathrm{AU}$.

Various sources of data are available : Levasseur-Regourd \& Dumont compilation of smoothed intensities (1980), Dumont \& Sanchez polarimetric measurements (1975), Fechtig, Leinert \& Grün compilation of intensity and polarimetric data (1981), and IRAS results (Hauser et al, 1984; Hauser \& Vrtilek, 1988).

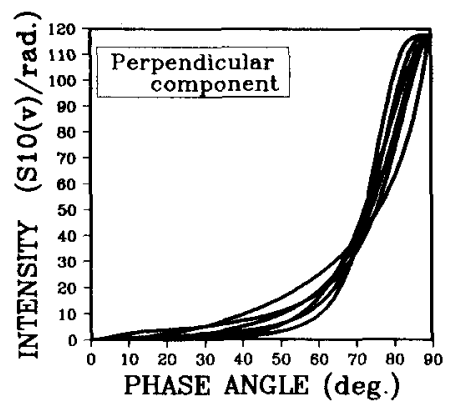

\subsection{DISCUSSION}

For the lines of sight with an ecliptic latitude varying from $70^{\circ}$ to $85^{\circ}$, the elevations obtained for the two positions of the node are stable (respectively 0.39 and $0.26 \mathrm{AU}$ ). The local values of the polarization are in excellent agreement (in average 0.08 at $h=0.39 \mathrm{AU}$, $\alpha=75^{\circ}$ and 0.16 at $\mathrm{h}=0.26 \mathrm{AU}, \alpha=68^{\circ}$ ), with an error lower than $5 \%$. It is possible to compare these results with those already found towards the symmetry pole $(0.08$ at $h=0.40$ 
$\mathrm{AU}, \alpha=68^{\circ}$, and 0.16 at $\mathrm{h}=0.27 \mathrm{AU}, \alpha=75^{\circ}$ ). The two sets of values are quite similar. Also, for $\beta=0^{\circ}$, there is a good agreement near the martian node with the tangential plane method results and the symmetry plane method results (respectively 0.08 at $R=1.51 \mathrm{AU}$, $\alpha=41.5^{\circ}$, and 0.06 at $R=1.54 \mathrm{AU}, \alpha=40.4^{\circ}$ ).

Gradients of intensity and temperature with solar distance are obtained (LevasseurRegourd, 1990), as well as a curve describing the evolution of local intensity with phase angle (Levasseur-Regourd et al., 1991). It is then possible to normalize the local contributions at constant phase angle and solar distance .

\section{Towards the Symmetry Pole}

Curves of integrated intensity and emissivity allow us to obtain, at the radial node position and at the four polar node positions, the values of local intensity, polarization degree, emissivity, temperature and albedo. Evolution of these values with elevation above the symmetry plane, between 0 and $0.40 \mathrm{AU}$, can be derived. Results are given at normalized phase angle and solar distance. From published IRAS data, local temperature is found to decrease significantly from $255 \mathrm{~K}$ in the symmetry plane to $230 \mathrm{~K}$ at $\mathrm{h}=0.40 \mathrm{AU}$ (with an relative error of the order of $5 \%$ ). Indeed, the local albedo is computed to increase from 0.08 at $\mathrm{h}=0 \mathrm{AU}$ to 0.10 at $\mathrm{h}=0.40 \mathrm{AU}$ (with a relative error of $5 \%$ ). Simultaneously, a decrease of the local polarization degree, from 0.29 in the symmetry plane to 0.18 at 0.40 $\mathrm{AU}$ above it, is found with a relative error smaller than $10 \%$ (fig. 3 ).

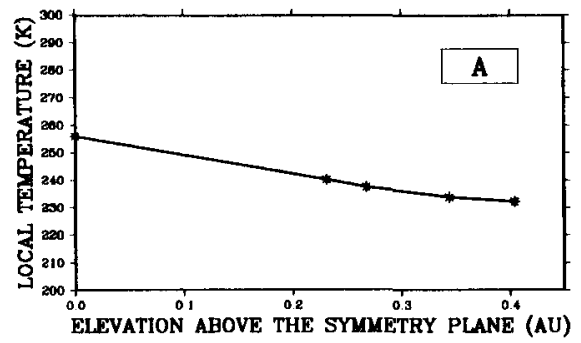

Figure 3 : Evolution with elevation towards the pole of the symmetry plane of local temperature (A), local polarization degree (B) and local albedo $(\mathrm{C})$. The values are normalized at $\mathrm{R}=1 \mathrm{AU}, \alpha=90^{\circ}$.
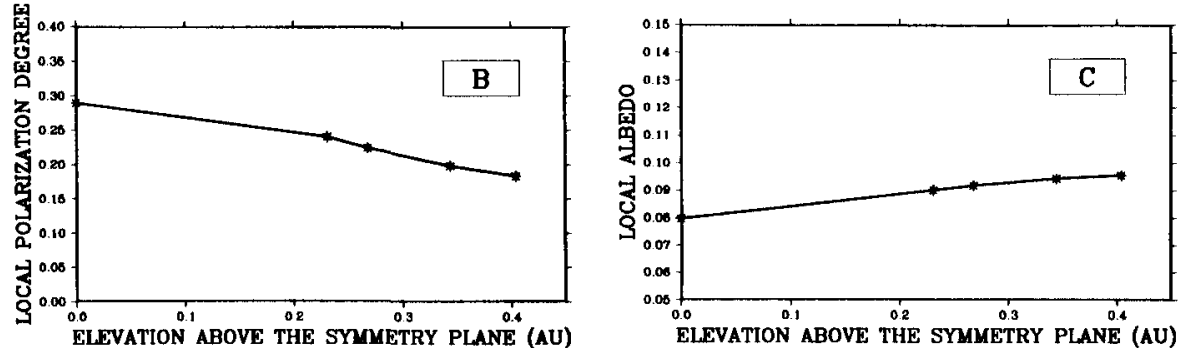

\section{In the Tangential Plane}

The tangential node positions correspond to an elevation, a solar distance, a Earth's distance and a phase angle, respectively between 0 and $0.40 \mathrm{AU}, 1.02$ and $1.74 \mathrm{AU}, 0$ and $1.5 \mathrm{AU}, 35^{\circ}$ and $75^{\circ}$. The evolution of the local polarization degree, at normalized solar distance and phase angle, can be presented in the tangential plane as a function of the elevation and the Earth's distance (fig.4). The average relative error is of the order of $10 \%$. The decrease of the polarization with elevation, previously found towards the symmetry 
pole, is here confirmed in the area of the tangential plane previously described. The decrease of the polarization seems to be continuous, but steeper between 0.2 and $0.4 \mathrm{AU}$ than between 0 to $0.2 \mathrm{AU}$ (fig.4).

Figure 4: Evolution of the local polarization degree with Earth's distance and elevation above the symmetry plane. Each dot represents the nodal region obtained after local inversion.

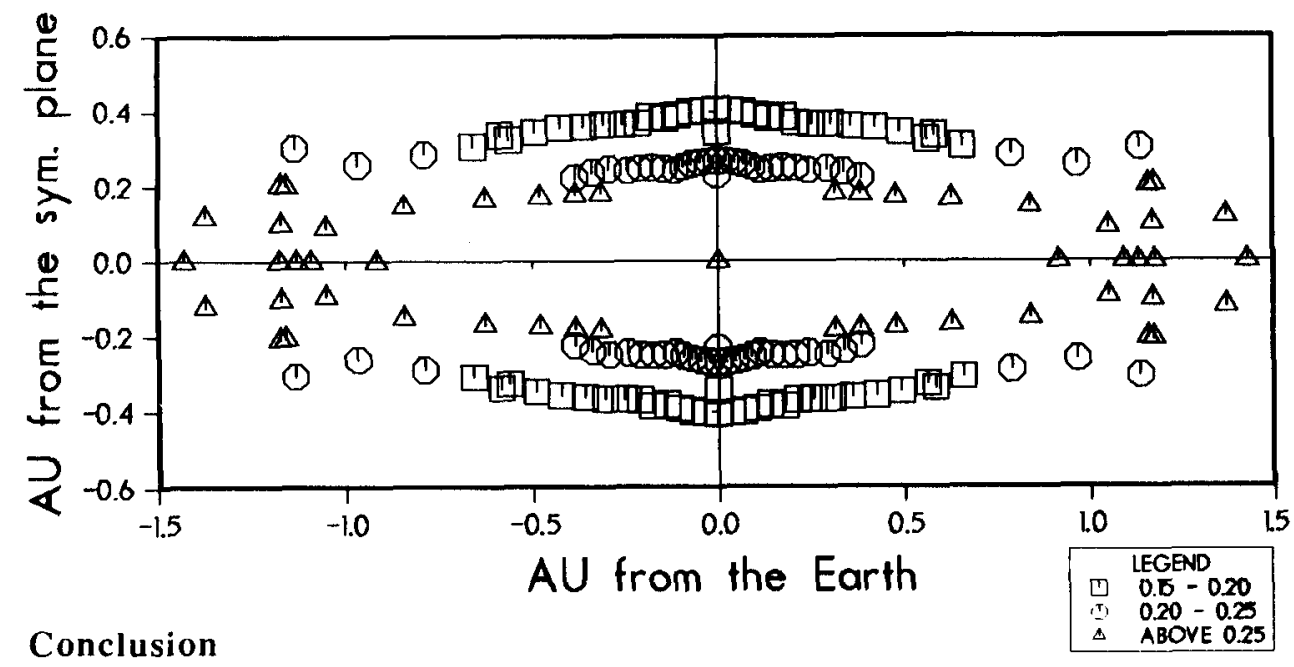

Local values of the optical properties of the grains, derived from measurements of integrated intensity and emissivity (lines of sight in the tangential plane), allow us to demonstrate the decrease of the local polarization degree with elevation. The evolution of the local physical properties with elevation confirms the heterogeneity of the zodiacal cloud out of its symmetry plane. The variations of the properties of the grains explain the differences between the various models (fitting visual or thermal observations) and suggest a dust population from different origins (Levasseur-Regourd et al., 1991).

\section{References}

Dumont R. (1983), Planet. Space Sci. 31 (12), 1381-1387.

Dumont R. \& Levasseur-Regourd A.C. (1985), Planet. Space Sci. 33 (1), 1-9.

Dumont R. \& Levasseur-Regourd A.C. (1988), Astron. Astrophys. 191, 154-160.

Dumont R. \& Sanchez F. (1975), Astron. Astrophys. 38, 405-412.

Fechtig H., Leinert C., \& Grün E. (1981), in K. Schaifers \& H. Voigt (eds.), LandoltBörnstein N.S., 2a, Springer-Verlag, Berlin, 228-243.

Hauser M.G., Gillett, F.C., Low F.L., Gautier T.N., Beichman C.A., Neugebauer G., Aumann H.H., Baud B., Boggess N., Emerson J.P., Houck J.R., Soifer B.T. \& Walker R.G. (1984), Astrophys. J. 278, L15-L18.

Hauser \& Vrtilek (1988), Private communication.

Levasseur-Regourd A.C. (1990), in B. Battrick (ed.), ESA SP 315, 105-111.

Levasseur-Regourd A.C. \& Dumont R. (1980), Astron. Astrophys., 38, 277-279.

Levasseur-Regourd A.C. \& Dumont R. (1990), Adv. Space. Res., 10, 3, 163-170.

Levasseur-Regourd A.C., Dumont R., \& Renard J.B. (1990), Icarus, 86, 264-272.

Levasseur-Regourd A.C., Renard J.B. \& Dumont R. (1991), this issue. 